\title{
Calculated Absorption and Scattering Properties of Gold Nanoparticles of Different Size, Shape, and Composition: Applications in Biological Imaging and Biomedicine
}

\author{
Prashant K. Jain, ${ }^{\dagger}$ Kyeong Seok Lee, ${ }^{\dagger}$ Ivan H. El-Sayed, $*, *$ and Mostafa A. El-Sayed $*, \dagger$ \\ Laser Dynamics Laboratory, School of Chemistry and Biochemistry, Georgia, Institute of Technology, \\ Atlanta, Georgia 30332-0400, and Department of Otolaryngology-Head and Neck Surgery, \\ Comprehensive Cancer Center, University of California at San Francisco, San Francisco, California 94143
}

Received: December 8, 2005; In Final Form: February 22, 2006

\begin{abstract}
The selection of nanoparticles for achieving efficient contrast for biological and cell imaging applications, as well as for photothermal therapeutic applications, is based on the optical properties of the nanoparticles. We use Mie theory and discrete dipole approximation method to calculate absorption and scattering efficiencies and optical resonance wavelengths for three commonly used classes of nanoparticles: gold nanospheres, silica-gold nanoshells, and gold nanorods. The calculated spectra clearly reflect the well-known dependence of nanoparticle optical properties viz. the resonance wavelength, the extinction cross-section, and the ratio of scattering to absorption, on the nanoparticle dimensions. A systematic quantitative study of the various trends is presented. By increasing the size of gold nanospheres from 20 to $80 \mathrm{~nm}$, the magnitude of extinction as well as the relative contribution of scattering to the extinction rapidly increases. Gold nanospheres in the size range commonly employed $(\sim 40 \mathrm{~nm})$ show an absorption cross-section 5 orders higher than conventional absorbing dyes, while the magnitude of light scattering by $80-\mathrm{nm}$ gold nanospheres is 5 orders higher than the light emission from strongly fluorescing dyes. The variation in the plasmon wavelength maximum of nanospheres, i.e., from $\sim 520$ to $550 \mathrm{~nm}$, is however too limited to be useful for in vivo applications. Gold nanoshells are found to have optical cross-sections comparable to and even higher than the nanospheres. Additionally, their optical resonances lie favorably in the near-infrared region. The resonance wavelength can be rapidly increased by either increasing the total nanoshell size or increasing the ratio of the core-toshell radius. The total extinction of nanoshells shows a linear dependence on their total size, however, it is independent of the core/shell radius ratio. The relative scattering contribution to the extinction can be rapidly increased by increasing the nanoshell size or decreasing the ratio of the core/shell radius. Gold nanorods show optical cross-sections comparable to nanospheres and nanoshells, however, at much smaller effective size. Their optical resonance can be linearly tuned across the near-infrared region by changing either the effective size or the aspect ratio of the nanorods. The total extinction as well as the relative scattering contribution increases rapidly with the effective size, however, they are independent of the aspect ratio. To compare the effectiveness of nanoparticles of different sizes for real biomedical applications, size-normalized optical cross-sections or per micron coefficients are calculated. Gold nanorods show per micron absorption and scattering coefficients that are an order of magnitude higher than those for nanoshells and nanospheres. While nanorods with a higher aspect ratio along with a smaller effective radius are the best photoabsorbing nanoparticles, the highest scattering contrast for imaging applications is obtained from nanorods of high aspect ratio with a larger effective radius.
\end{abstract}

\section{Introduction}

The strongly enhanced surface plasmon resonance of noble metal nanoparticles at optical frequencies makes them excellent scatterers and absorbers of visible light. ${ }^{1-3}$ Superior optical properties, coupled with recent advances in nanoparticle synthesis, ${ }^{4}$ conjugation, ${ }^{5}$ and assembly, ${ }^{6}$ have stimulated interest in the use of plasmon-resonant nanoparticles

* Corresponding authors. E-mail: Mostafa A. El-Sayed: mostafa. el-sayed@chemistry.gatech.edu (M.A.E.); ielsayed@ohns.ucsf.edu (I.H.E.). Telephone: 404-894-0292 (M.A.E.); 415-353-2401 (I.H.E.). Fax: 404-8940294 (M.A.E.); Fax: 415-353-2603 (I.H.E.)

Georgia Institute of Technology.

$\doteqdot$ University of California at San Francisco. and nanostructures for optical and photonic applications s $^{7,8}$ and, more recently, for biomedical applications. ${ }^{9-26}$ Nanoparticles composed of gold offer, in addition to their enhanced absorption and scattering, good biocompatibility, facile synthesis, ${ }^{4}$ and conjugation to a variety of biomolecular ligands, antibodies, and other targeting moieties, ${ }^{5}$ making them suitable for use in biochemical sensing and detection, ${ }^{9-11}$ medical diagnostics, and therapeutic applications. ${ }^{12,13}$ There have been several demonstrations of bioaffinity sensors based on the plasmon absorption and scattering of nanoparticles ${ }^{9,10}$ and their assemblies. ${ }^{11}$

Another notable use of gold nanoparticles has been as contrast agents in cellular and biological imaging. ${ }^{14-17}$ Contrast 
agents in medical and biological imaging ${ }^{18}$ improve the sensitivity and diagnostic ability of the imaging modality by sitespecifically labeling tissues or cells of interest. Cellular imaging utilizing microscopy techniques and immunotargeted optical contrast agents provides anatomic details of cells and tissue architecture important for diagnosis of cancer as well as other disorders. Biomedical imaging contrast agents have been traditionally based on photoabsorbing and fluorescent dyes such as malachite green and rhodamine-6G. ${ }^{19}$ More recently, quantum dots have been used and studied for biological and cell imaging due to their unique size-dependent fluorescence properties. ${ }^{20,21}$ However, the potential human toxicity and cytotoxicity of the semiconductor material are two major problems for its in vitro and in vivo application. Colloidal gold nanoparticles have become an important alternative as imaging agents due to their potential noncytotoxic, facile immunotargeting ${ }^{5}$ as well as due to their nonsusceptibility to photobleaching or chemical/thermal denaturation, a problem commonly associated with dyes. ${ }^{22}$ Immunogold nanoparticles conjugated to antibodies have been widely used for biological labeling and staining for electron microscopy. ${ }^{23}$ Recently, strongly absorbing nanoparticles composed of gold have been shown to offer excellent promise for cell and tissue imaging by using techniques such as multiphoton plasmon resonance microscopy ${ }^{14}$ and photoacoustic tomography. ${ }^{15}$ Similarly, the strong light scattering of gold nanoparticles has been exploited for real-time optical imaging of precancer by using confocal reflectance microscopy. ${ }^{16}$ El-Sayed et al. have demonstrated differentiation of cancerous cells from noncancerous cells by dark field light-scattering imaging and absorption spectroscopy of solid $\sim 40 \mathrm{~nm}$ gold nanospheres immunotargeted to EGFR overexpressed on cancer cells. ${ }^{17}$ Along with cancer imaging and diagnostic applications, the ability of gold nanoparticles to efficiently convert absorbed light into localized heat can be readily employed for therapy based on photothermal destruction of cancerous cells. ${ }^{24-28}$ For example, Hirsch et al. ${ }^{24}$ employed NIR absorbing silica-gold core-shell particles for photothermal destruction of human breast carcinoma cells in vitro as well as solid tumors in vivo. Recently, Loo et al. ${ }^{25}$ reported simultaneous imaging and therapy of breast cancers in vitro using silica-gold nanoshells that were conjugated with anti-Her2 antibodies. El-Sayed et al. and Huang et al. used immunotargeted nanospheres of solid gold for imaging and selective photothermal destruction of cancer cells by Ar laser irradiation. ${ }^{26,27}$ More recently, Huang et al. provided an in vitro demonstration of gold nanorods conjugated to antiEGFR antibodies as novel contrast agents for both NIR cell imaging and photothermal cancer therapy. ${ }^{28}$

The effectiveness of nanoparticles as biomedical imaging contrast and therapeutic agents depends on their optical properties. For instance, a high-scattering cross-section is essential for cell imaging applications based on light-scattering microscopy. On the other hand, effective photothermal therapy with minimal laser dosage requires a high nanoparticle absorption cross-section with low scattering losses. Biosensing applications based on surface plasmon resonance shifts necessitate strong resonance in the wavelength sensitivity range of the instrument as well as narrow optical resonance line widths. ${ }^{29}$ For actual in vivo imaging and therapeutic applications, the optical resonance of the nanoparticles is strongly desired to be in the near-infrared (NIR) region of the biological water window, where the tissue transmissivity is the highest. ${ }^{30}$ In addition, the nanoparticle size is also an important consideration for nanoparticle uptake and retention by cells and tissue. ${ }^{31}$
It is well-known that the plasmon resonance of metal nanoparticles is strongly sensitive to the nanoparticle size, shape, and the dielectric properties of the surrounding medium. Optical properties of gold nanoparticles can thus be readily

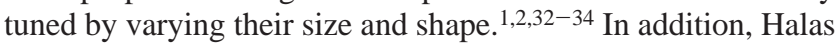
and co-workers have shown that the use of composite nanoparticles based on a core-shell morphology (e.g., silica-gold nanoshells) allows optical tunability by variation in the composition. ${ }^{35}$

There have been several experimental reports ${ }^{2,36}$ on the optical properties of metal nanoparticles, including gold nanospheres, ${ }^{33,34,36-42}$ nanorods, ${ }^{33,34,36,43}$ and nanoprisms, ${ }^{44}$ silver nanospheres, ${ }^{36,37,39,45-47}$ nanowires ${ }^{48}$ and nanoprisms,${ }^{49-52}$ copper nanospheres, ${ }^{37,45,53}$ aluminum nanospheres, ${ }^{36}$ bimetallic nanoparticles, ${ }^{54,55}$ composite nanoparticles with a core-shell structure, ${ }^{35,56-60}$ and nanoparticle chains and assemblies. ${ }^{61-63}$ At the same time, well-established theoretical tools based on the Mie theory ${ }^{64}$ and the discrete dipole approximation (DDA) ${ }^{65}$ method have been readily exploited for a quantitative study of the nanoparticle optical properties of different size, shape, composition, and aggregation state, etc. ${ }^{2,36,66-73}$ In this paper, we use Mie theory and the DDA method to calculate the absorption and scattering efficiencies and optical resonance wavelengths of gold nanospheres, silica-gold nanoshells, and gold nanorods, for various nanoparticle dimensions, so as to aid the selection of nanoparticles for specific biomedical applications. The calculated optical cross-sections of all three nanoparticle classes are found to be a few orders of magnitude higher than those for conventionally used absorbing and fluorescent dyes. Besides, the optical properties of nanoparticles, i.e., the optical resonance wavelength, the extinction cross-section, and the relative contribution of scattering to the extinction, are strongly dependent on the nanoparticle dimensions, allowing tunability for specific applications. For all three nanoparticle types, the increase in the size results in an increase in the extinction as well as the relative contribution of scattering. Nanospheres offer resonance wavelengths in the visible region, however, the tunability of the wavelength with size is too limited to be useful for in vivo biomedical applications. In the case of nanoshells, the resonance wavelength can be rapidly tuned in the NIR region by either changing the total nanoshell size or the ratio of the core/shell radius. Similarly, the optical resonance of nanorods can be linearly tuned across the near-infrared region by changing either the effective size or the aspect ratio of the nanorods. To compare the effectiveness of nanoparticles of different size for real-life biomedical applications, sizenormalized optical cross-sections or per micron coefficients have been calculated. Gold nanorods on account of an order of magnitude higher per micron absorption and scattering coefficients, combined with easy resonance tunability and lack of cytotoxicity, are concluded to offer the best imaging contrast as well as the highest effectiveness for photothermal therapy applications.

\section{Calculation Method}

The optical properties of gold nanospheres, nanorods, and silica-gold nanoshells were quantified in terms of their calculated absorption and scattering efficiency ( $Q_{\text {abs }}$ and $\left.Q_{\text {sca }}\right)$ and their optical resonance wavelength $\left(\lambda_{\max }\right)$.

Gold Nanospheres and Silica-Gold Nanoshells. For nanospheres of gold, $Q_{\text {abs }}$ and $Q_{\text {sca }}$ were calculated on the basis of Mie theory for homogeneous spheres. ${ }^{64}$ The Mie total extinction and scattering efficiency $Q_{\text {ext }}$ and $Q_{\text {sca }}$ for a homogeneous sphere are expressed as infinite series: 


$$
\begin{gathered}
Q_{\mathrm{ext}}=\frac{2}{x^{2}} \sum_{n=1}^{\infty}(2 n+1) \operatorname{Re}\left[a_{n}+b_{n}\right] \\
Q_{\mathrm{sca}}=\frac{2}{x^{2}} \sum_{n=1}^{\infty}(2 n+1)\left[a_{n}^{2}+b_{n}^{2}\right] \\
Q_{\mathrm{abs}}=Q_{\mathrm{ext}}-Q_{\mathrm{sca}} \\
a_{n}=\frac{m \psi_{n}(m x) \psi_{n}^{\prime}(x)-\psi_{n}(x) y_{n}^{\prime}(m x)}{m \psi_{n}(m x) \xi_{n}^{\prime}(x)-m \xi_{n}(x) \psi_{n}^{\prime}(m x)} \\
b_{n}=\frac{\psi_{n}(m x) \psi_{n}^{\prime}(x)-m \psi_{n}(x) \psi_{n}^{\prime}(m x)}{\psi_{n}(m x) \xi_{n}^{\prime}(x)-m \xi_{n}(x) \psi_{n}^{\prime}(m x)}
\end{gathered}
$$

where $m$ is the ratio of refractive index of the sphere $n$ to that of the surrounding medium $n_{m}, x$ is the size parameter given as $2 \pi n_{m} R / \lambda, \psi_{n}$ and $\xi_{n}$ are the Riccati-Bessel functions, and the prime represents first differentiation with respect to the argument in parentheses. Numerical calculations of the Mie series were performed at discrete points in the wavelength range from 300 to $800 \mathrm{~nm}$.

Calculations of the optical absorption and scattering efficiency of silica-gold nanoshells were performed by using a computer code employing Mie scattering for concentric sphere geometry developed by Ivan Charamisinau. ${ }^{74}$ The required parameters for the code were the value of the core and shell radii, $R_{1}$ and $R_{2}$, and the complex refractive indices for the core, shell, and the surrounding medium, $n_{c}, n_{s}$, and $n_{m}$, respectively. $n_{c}$ was taken to be $1.44+0 i$ for the silica core at all wavelengths.

For gold, values of the complex dielectric function at different wavelengths were obtained from Johnson and Christy ${ }^{75}$ and corrected for nanoparticle size. ${ }^{76,77}$ Cubic interpolation was used to calculate the complex refractive indices at intermediate wavelengths, where data was not available directly from Johnson and Christy. The embedding medium for both nanospheres and nanoshells was considered to be water with a refractive index $n_{m}$ of $1.33+0 i$. The results of the Mie code for core-shell particles were checked against the Mie theory results for homogeneous spheres for three cases: vanishing shell, vanishing core, and vanishing refractive index difference between core and shell materials. There was excellent agreement in the calculated $Q_{\mathrm{abs}}$ and $Q_{\text {sca }}$ by the two methods, verifying the accuracy of the Mie code for core-shell particles.

In the case of nanospheres, the diameter $D$ of the particles is the only size variable. Mie theory calculations were performed for three different sizes, i.e., $D=20,40$, and $80 \mathrm{~nm}$, corresponding to the size range of gold nanospheres used in earlier demonstrations of light-scattering imaging ${ }^{16,17}$ and selective photothermal destruction of cancer cells. ${ }^{26,27}$ Mie parameters were also evaluated for 300-nm diameter polystyrene nanospheres, so as to serve as a basis for comparison of the optical properties of the metal nanoparticles. A refractive index value of $1.56+0 i$ was used for polystyrene at all wavelengths. Nanoshells, on the other hand, can be defined by two distinct variables: the total particle radius $R_{2}$ and the ratio of the core radius to the shell radius $R_{1} / R_{2}$. Calculations for nanoshells were performed for two different cases: first, at a fixed $R_{2}$ of $70 \mathrm{~nm}$ and $R_{1}$ varying as 40,50 , and $60 \mathrm{~nm}$, and second, for a fixed $R_{1} / R_{2} \sim 0.857$ and $R_{2}=70,105$, and $140 \mathrm{~nm}$.

Gold Nanorods. The calculation of the optical extinction, absorption, and scattering efficiency of gold nanorods was performed by using the discrete dipole approximation (DDA) method, ${ }^{65}$ which has been regarded as one of the most powerful and flexible electrodynamic methods for computing the optical properties of particles with an arbitrary geometry. For this calculation, we adopted the DDA code developed by Draine and Flatau ${ }^{65}$ and characterized the gold nanorod case with fixed target orientation, where the propagation direction of the incident light was assumed to be perpendicular to the optic axis of the nanorod. Only two orthogonal polarizations of incident light were considered in the calculation, one with an electric field parallel to the optic axis and another that is perpendicular to it. The gold nanorod was considered to have the geometry of a cylinder capped with two hemispheres. It is common to specify the size of a particle of an arbitrary shape and volume $V$ in terms of an effective radius given by:

$$
r_{\text {eff }}=(3 V / 4 \pi)^{1 / 3}
$$

which represents the radius of a sphere having a volume equal to that of the particle. Thus $r_{\text {eff }}$ defines the volume of the nanorod. An additional defining size variable in case of nanorods is the aspect ratio $(R)$, i.e., the ratio of the nanorod dimension along the long axis to that along the short axis. The calculations were therefore carried out for two different cases, one for nanorods with a fixed effective radius (and hence volume) $r_{\text {eff }}$ $=11.43 \mathrm{~nm}$ but different aspect ratios of 3.1,3.9, and 4.6 and the other for nanorods with a fixed $R$ of 3.9 but different $r_{\text {eff }}$ of $8.74,11.43,17.9$, and $21.86 \mathrm{~nm}$. Dielectric constants for gold at different wavelengths were assumed to be the same as that of the bulk metal. ${ }^{75}$ The refractive index of the surrounding medium was considered to be $1.34+0 i$ at all wavelengths, close to that of water. Details of the DDA calculations for nanorods have been described elsewhere. ${ }^{68}$

\section{Results and Discussion}

Gold Nanospheres. Figure 1 shows the calculated spectra of the efficiency of absorption $Q_{\mathrm{abs}}$, scattering $Q_{\text {sca }}$, and extinction $Q_{\text {ext }}$ for gold nanospheres $(D=20,40$, and $80 \mathrm{~nm})$ and polystyrene nanospheres $(D=300 \mathrm{~nm})$. The dimensionless efficiencies $Q_{\mathrm{abs}}, Q_{\text {sca }}$, and $Q_{\text {ext }}$ can be converted to the corresponding cross-sections $C_{\mathrm{abs}}, C_{\mathrm{sca}}$, and $C_{\mathrm{ext}}$ by multiplication with the cross-sectional area of the nanoparticle. $C_{\mathrm{abs}}, C_{\mathrm{sca}}$, and $C_{\text {ext }}$ have units of $\mathrm{m}^{2}$ because they represent an equivalent cross-sectional area of the particle that contributes to the absorption, scattering, and extinction of the incident light. The cross-sections can also be directly related to the molar coefficients measured by spectrophotometry.

It is seen that the optical cross-sections of the gold nanospheres are typically 4-5 orders of magnitude higher compared to those of conventionally used dyes. For instance, gold nanospheres with a diameter of $40 \mathrm{~nm}$, which have been successfully used by El-Sayed et al. ${ }^{26}$ and Huang et al. ${ }^{27}$ for laser photothermal destruction of cancer cells, have a calculated absorption cross-section of $2.93 \times 10^{-15} \mathrm{~m}^{2}$ (thus corresponding to a molar absorption coefficient $\epsilon$ of $\left.7.66 \times 10^{9} \mathrm{M}^{-1} \mathrm{~cm}^{-1}\right)^{78}$ at a plasmon resonance wavelength maximum $\lambda_{\max }$ of $528 \mathrm{~nm}$. This value is 5 orders larger than the molar extinction coefficient for indocyanine green $\left(\epsilon=1.08 \times 10^{4} \mathrm{M}^{-1} \mathrm{~cm}^{-1}\right.$ at $\left.778 \mathrm{~nm}^{79}\right)$, a NIR dye commonly used in laser photothermal tumor therapy. ${ }^{80,81}$ Similarly other strongly absorbing dyes such as rhodamine-6G $\left(\epsilon=1.16 \times 10^{5} \mathrm{M}^{-1} \mathrm{~cm}^{-1} \text { at } 530 \mathrm{~nm}\right)^{82}$ and malachite green $\left(\epsilon=1.49 \times 10^{5} \mathrm{M}^{-1} \mathrm{~cm}^{-1} \text { at } 617 \mathrm{~nm}\right)^{82}$ have 4 orders much lower absorption as compared to the nanoparticles.

In addition, the magnitude of visible light scattering by the metal nanoparticles $\left(C_{\mathrm{sca}}=1.23 \times 10^{-14} \mathrm{~m}^{2}\right.$ at $560 \mathrm{~nm}$ for 

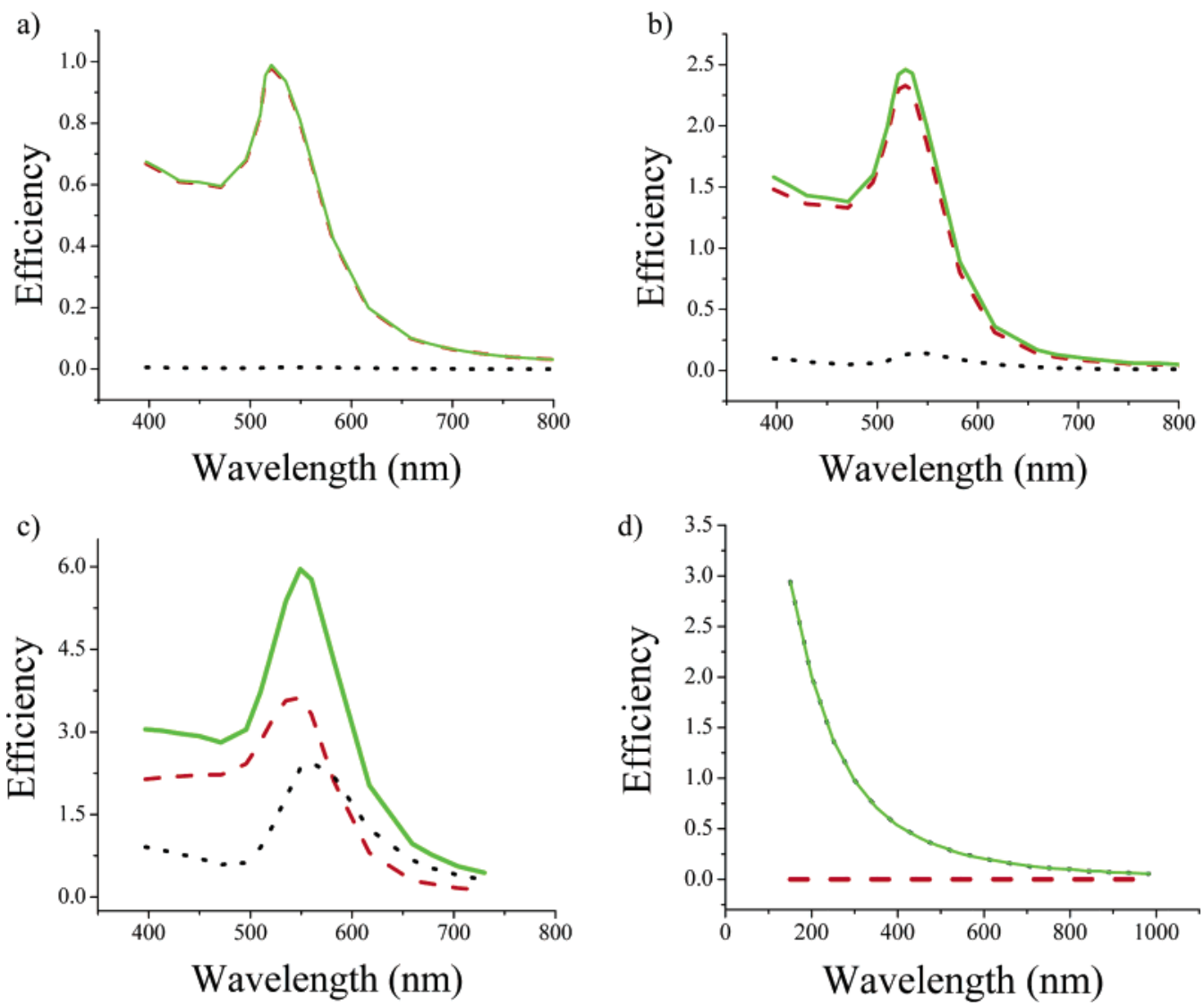

Figure 1. Calculated spectra of the efficiency of absorption $Q_{\text {abs }}$ (red dashed), scattering $Q_{\text {sca }}$ (black dotted), and extinction $Q_{\text {ext }}$ (green solid) for gold nanospheres (a) $D=20 \mathrm{~nm}$, (b) $D=40 \mathrm{~nm}$, (c) $D=80 \mathrm{~nm}$, and polystyrene nanospheres (d) $D=300 \mathrm{~nm}$.

80 -nm gold nanospheres) is comparable to the scattering from the much larger $300-\mathrm{nm}$ polystyrene nanospheres $\left(C_{\mathrm{sca}}=1.77\right.$ $\times 10^{-14}$ at $560 \mathrm{~nm}$ ), which are commonly used in confocal imaging of cells. ${ }^{83}$ The light emission from fluorescent molecules such as fluorescein $\left(\epsilon=9.23 \times 10^{4} \mathrm{M}^{-1} \mathrm{~cm}^{-1}\right.$ with a quantum yield $\sim 0.98$ at $483 \mathrm{~nm}),{ }^{82}$ also commonly used in imaging is 5 orders of magnitude lower than the light scattering from the $80-\mathrm{nm}$ gold nanospheres $\left(C_{\text {sca }}=1.23 \times 10^{-14} \mathrm{~m}^{2}\right.$ corresponding to a molar scattering coefficient of $3.22 \times 10^{10}$ $\mathrm{M}^{-1} \mathrm{~cm}^{-1}$ ). The superior scattering properties of gold nanospheres have already been exploited for the selective imaging of cancer cells by using simple dark field microscopy ${ }^{17}$ and confocal microscopy. ${ }^{16}$ The strongly enhanced absorption and scattering of metal nanoparticles as compared to polystyrene nanospheres or dyes is attributable to the well-known surface plasmon oscillation of electrons of the metal nanoparticle. ${ }^{2,36}$

Silica-Gold Nanoshells. Calculated spectra of $Q_{\text {abs }}, Q_{\text {sca }}$, and $Q_{\text {ext }}$ for various dimensions of the silica-gold nanoshells (i.e., $R_{1}=40 \mathrm{~nm} R_{2}=70 \mathrm{~nm}, R_{1}=50 \mathrm{~nm} R_{2}=70 \mathrm{~nm}, R_{1}=60$ $\mathrm{nm} R_{2}=70 \mathrm{~nm}, R_{1}=90 \mathrm{~nm} R_{2}=105 \mathrm{~nm}, R_{1}=120 \mathrm{~nm} R_{2}$ $=140 \mathrm{~nm}$, and $R_{1}=120 \mathrm{~nm} R_{2}=155 \mathrm{~nm}$ ) are shown in Figure 2 . The nanoshells show absorption and scattering cross-sections $\left(C_{\text {abs }}=5.09 \times 10^{-14} \mathrm{~m}^{2}, C_{\mathrm{sca}}=3.25 \times 10^{-14}\right.$ for $R_{1}=60 \mathrm{~nm}$ $R_{2}=70 \mathrm{~nm}$ nanoshell) that are comparable to and even higher in magnitude than those of solid gold nanospheres. Additionally, the nanoshell optical resonance lies in the NIR region $\left(\lambda_{\max }=\right.$ $892 \mathrm{~nm}$ for $R_{1}=60 \mathrm{~nm} R_{2}=70 \mathrm{~nm}$ ), where biological tissue transmissivity is the highest ${ }^{30}$ and away from the hemoglobin visible absorption around 500-600 nm. ${ }^{84}$ Thus the nanoshells are much more suited to in vivo imaging and therapy applications as compared to the gold nanospheres. Silica-gold nanoshells have been successfully employed in experimental demonstrations by Hirsch et al. ${ }^{24}$ and Loo et al. ${ }^{25}$ However, there have been concerns about the potential carcinogenicity of the silica material of the nanoshell core. ${ }^{85}$ It would thus be highly desirable to have nanoparticles of solid gold with NIR absorption. However, as seen in Figure 4a, change in the nanosphere size does not provide the desired tunability in the optical resonance. In fact, it is known that pure gold nanospheres have resonance around $528 \mathrm{~nm}$ for different sizes from tens to 100 $\mathrm{nm} .{ }^{33}$

Gold Nanorods. It is well-known that, by changing the shape of nanoparticles to that of elongated rods, the optical characteristics can be significantly changed. $2,33,34,36,43,73$ Gold nanorods possess, in addition to the surface plasmon band around 528 $\mathrm{nm}$ seen in gold nanospheres, a band at longer wavelengths due to the plasmon oscillation of electrons along the long axis of the nanorods..$^{2,33,34,36,43,73}$ The calculated absorption, scattering, and extinction spectra of the surface plasmon band of gold nanorods have been shown in Figure 3. Figure 3a shows calculations for nanorods with a fixed aspect ratio $R$ of 3.9 and effective radius $r_{\mathrm{eff}}=8.74,11.43,17.90$, and $21.86 \mathrm{~nm}$. Calculations for a fixed $r_{\text {eff }}$ (and hence volume) of $11.43 \mathrm{~nm}$ but with different aspect ratios $R=3.1,3.9$ and 4.6 are shown in Figure $3 b$. Thus the figure shows that the plasmon maximum of the nanorods (corresponding to the mode with the electric field parallel to the long axis of the nanorod) lie in the desirable NIR region, thus making gold nanorods potentially useful for in vivo applications. The magnitude of their NIR absorption and scattering $\left(C_{\mathrm{abs}}=1.97 \times 10^{-14} \mathrm{~m}^{2}\right.$ and $C_{\mathrm{sca}}=1.07 \times 10^{-14}$ at $\lambda_{\max }=842 \mathrm{~nm}$ for nanorods with $r_{\mathrm{eff}}=21.86 \mathrm{~nm}, R=3.9$ ) is comparable to that of the nanospheres and nanoshells, at a much smaller size or volume.

Optical Tunability in Nanoparticles. The calculated spectra for different nanoparticle types clearly reflect the well-known fact $^{2,33,34,36}$ that the surface plasmon resonance wavelength as 
a)

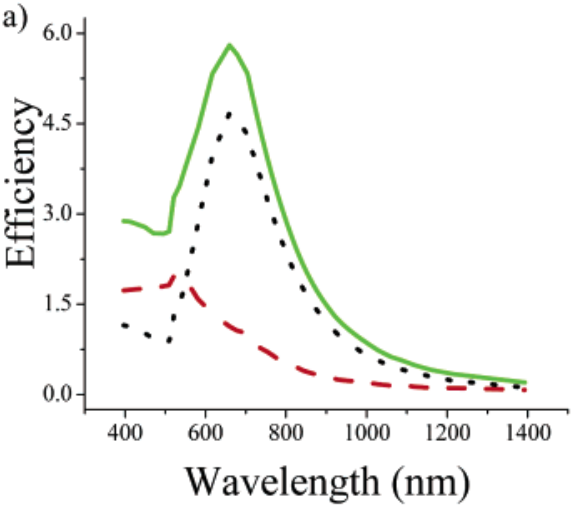

c)

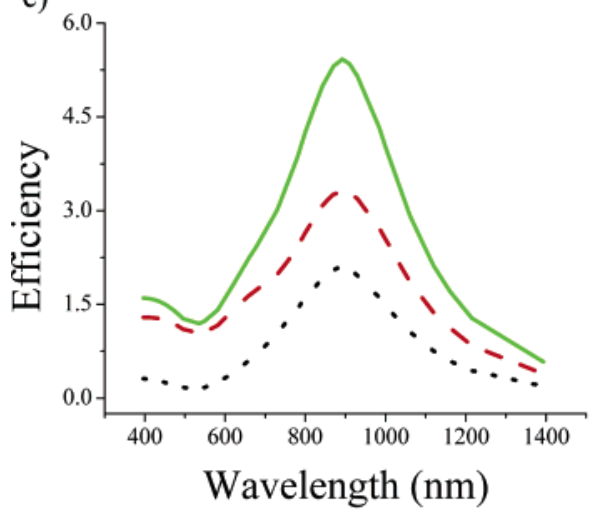

e)

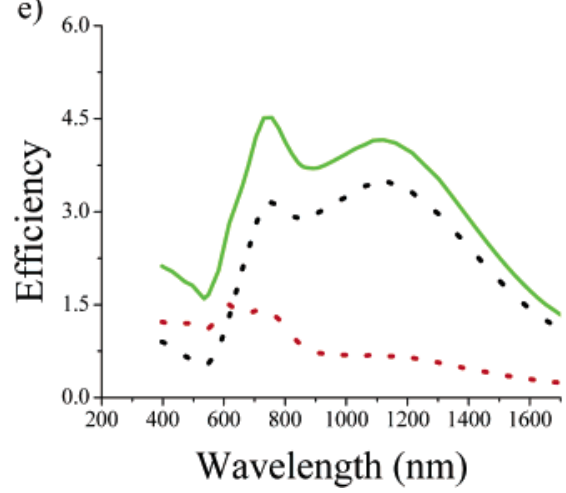

b)

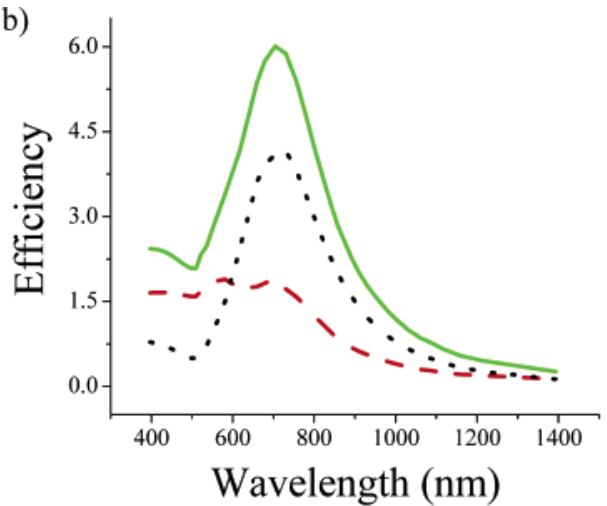

d)

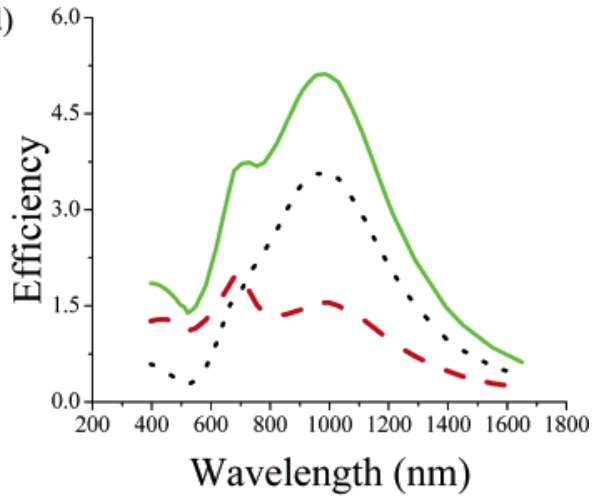

f)

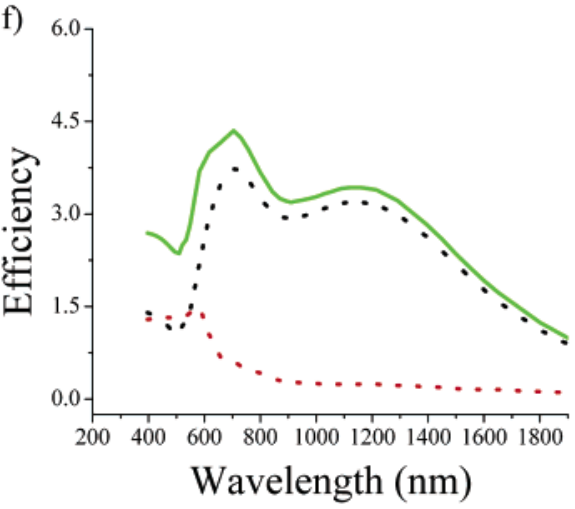

Figure 2. Calculated spectra of the efficiency of absorption $Q_{\text {abs }}$ (red dashed), scattering $Q_{\text {sca }}$ (black dotted), and extinction $Q_{\text {ext }}$ (green solid) for silica-gold nanoshells with dimensions (a) $R_{1}=40 \mathrm{~nm}, R_{2}=70 \mathrm{~nm}$, (b) $R_{1}=50 \mathrm{~nm}, R_{2}=70 \mathrm{~nm}$, (c) $R_{1}=60 \mathrm{~nm}, R_{2}=70 \mathrm{~nm}$, (d) $R_{1}=90 \mathrm{~nm}$, $R_{2}=105 \mathrm{~nm}$, (e) $R_{1}=120 \mathrm{~nm}, R_{2}=140 \mathrm{~nm}$, and (f) $R_{1}=120 \mathrm{~nm}, R_{2}=155 \mathrm{~nm}$.

well as the extent of the plasmon enhancement is highly dependent on the size, shape, and core-shell composition of the nanoparticles, thus allowing easy optical tunability, which is lacking in the case of dyes. To aid the selection of an appropriate nanoparticle for a suitable biomedical application, a systematic quantitative discussion of the trends in the optical tunability of nanoparticles follows.

Dependence of the Plasmon Resonance Maximum on Nanoparticle Dimensions. Figure 4 summarizes the dependence of the nanoparticle plasmon resonance wavelength maximum $\lambda_{\max }$ on the nanoparticle dimensions. Figure $4 \mathrm{a}$ shows the plot of $\lambda_{\max }$ versus the nanosphere diameter $D$. With increase in the nanosphere diameter from 20 to $80 \mathrm{~nm}$, there is a small redshift in the $\lambda_{\max }$ from $\sim 520$ to $550 \mathrm{~nm}$. Similar red-shift has been observed in the measured optical spectra of gold nanoparticles and is attributed to the effect of electromagnetic retardation in larger nanoparticles. 2,34,36,38,39,66,86 Nevertheless, changing the diameter $D$ of the nanospheres does not offer sufficient change in the surface plasmon resonance maximum to be useful in the present applications.

On the other hand, the optical resonance wavelength of nanoshells can be easily tuned by variation in their dimensions. As shown in Figure $4 \mathrm{~b}$, the nanoshell $\lambda_{\max }$ can be increased by increasing the total nanoshell radius $R_{2}$ while keeping $R_{1} / \mathrm{R}_{2}$ fixed. Alternatively, Figure $4 \mathrm{c}$ shows that the $\lambda_{\max }$ can be tuned by changing the relative core-shell dimensions $R_{1} / \mathrm{R}_{2}$ at a fixed total nanoshell size $R_{2}$. In other words, reducing the shell thickness shifts $\lambda_{\max }$ to longer wavelengths. In the case of larger nanoshells, the spectra show additional resonance peaks having strong intensity at shorter wavelengths as compared to the dipolar plasmon resonance. For example, for the nanoshell configuration with $R_{1}=120 \mathrm{~nm} R_{2}=140 \mathrm{~nm}$, a resonance peak can be seen around $756 \mathrm{~nm}$ in addition to the dipolar band at $1120 \mathrm{~nm}$ (Figure 2e). An additional resonance can also be seen in the case of $R_{1}=120 \mathrm{~nm} R_{2}=155$ (Figure 2f). These additional resonances arise from quadrupolar oscillations in the 

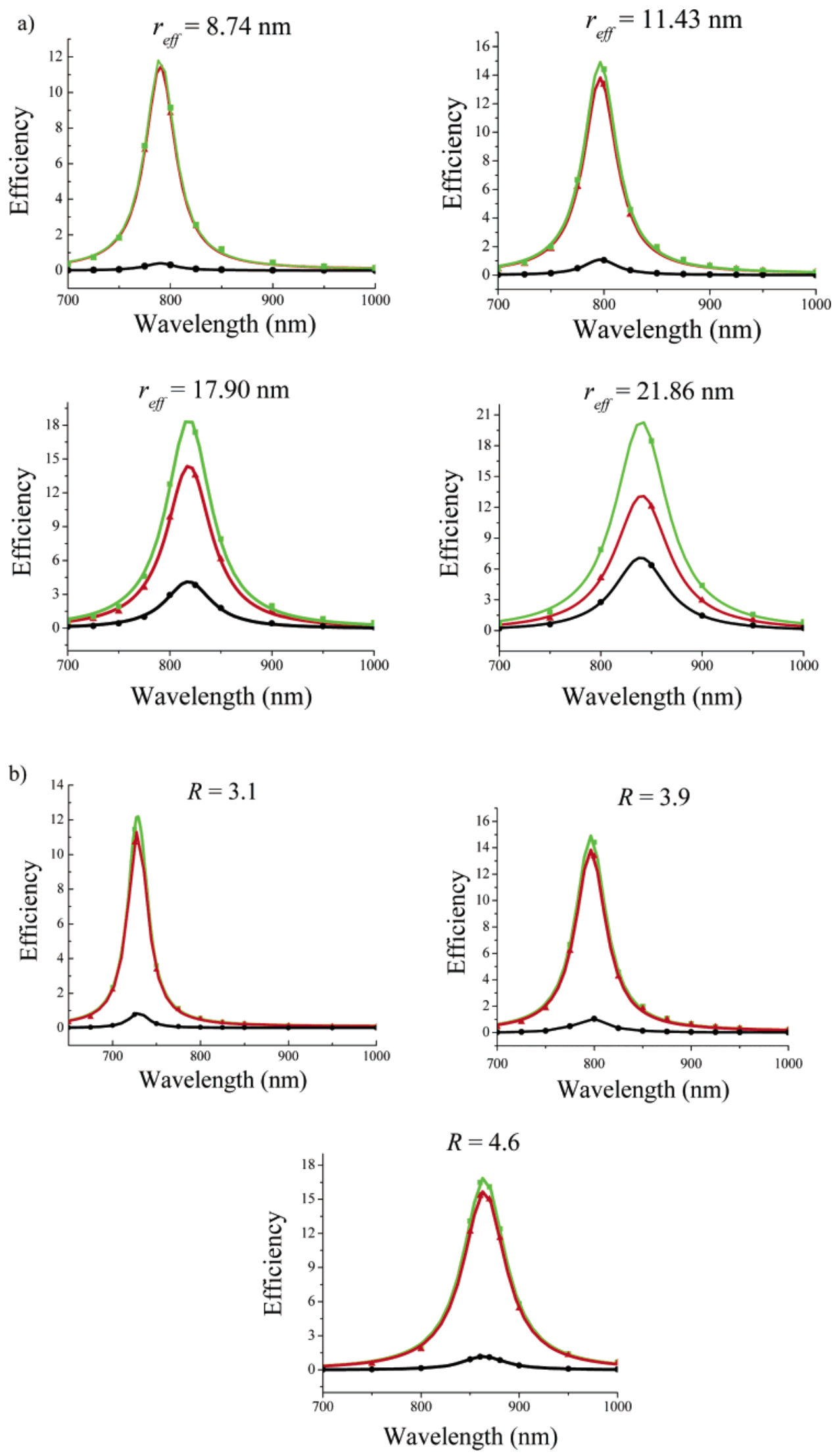

Figure 3. Calculated spectra of the efficiency of absorption $Q_{\mathrm{abs}}$ (red triangles), scattering $Q_{\text {sca }}$ (black circles), and extinction $Q_{\mathrm{ext}}$ (green squares) for gold nanorods (a) with fixed aspect ratio $R=3.9$ and $r_{\text {eff }}=8.74,11.43,17.90$, and $21.86 \mathrm{~nm}$, and (b) with fixed effective radius $r_{\mathrm{eff}}=11.43$ $\mathrm{nm}$ and $R=3.1,3.9$, and 4.6. Note the solid curves are Lorentzian fits to the calculated data points.

nanoshells of larger size (comparable to the light wavelength) Similar multipolar excitation has been observed in the theoretical spectra of large triangular nanoprisms of gold calculated by
Shuford et al. ${ }^{69}$ in which case three distinct peaks corresponding to the multipole orders $l=1,2,3$ were seen at progressively shorter wavelengths. Multipolar resonance peaks in the nanoshells 

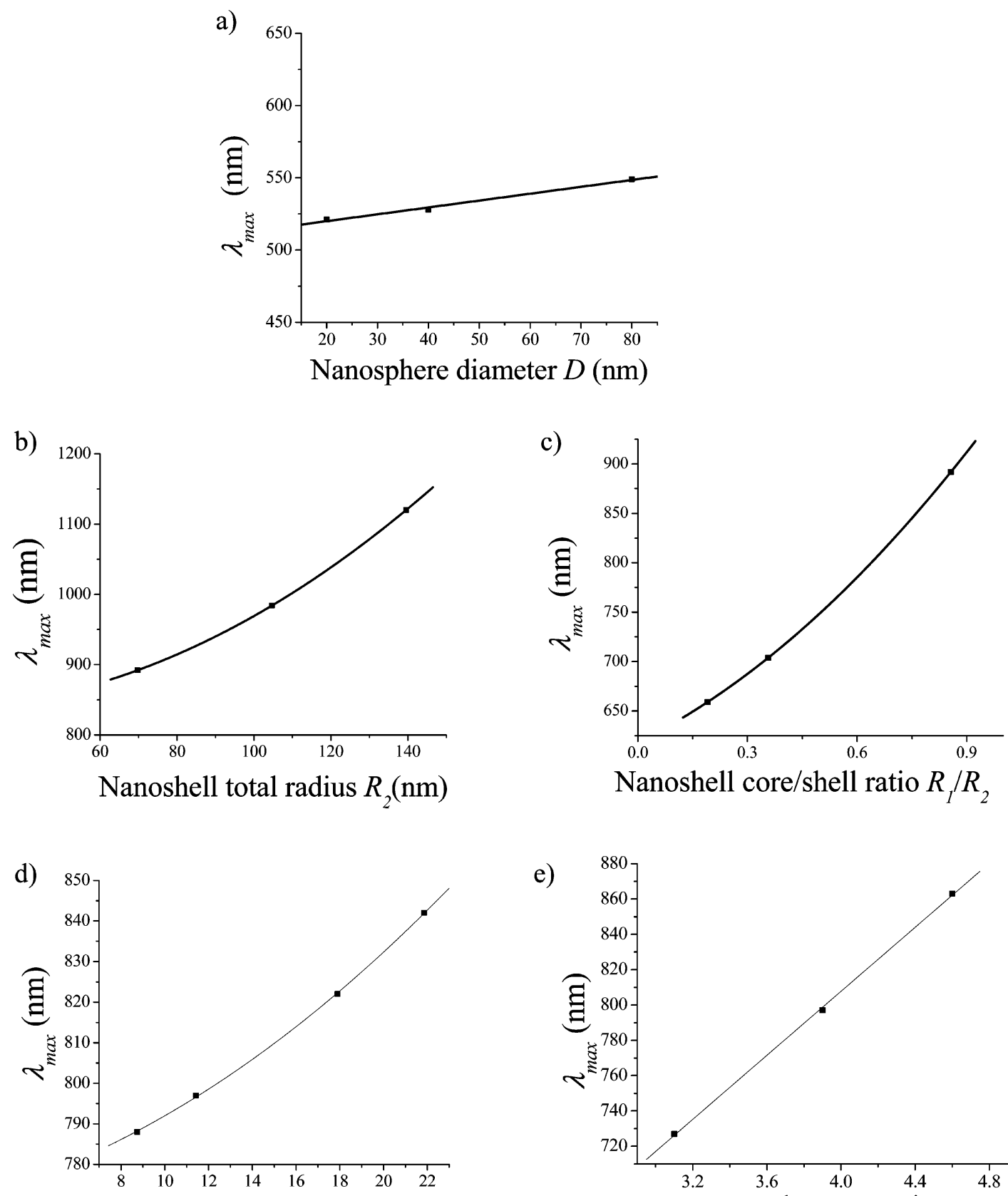

\section{Effective nanorod radius $r_{\text {eff }}(\mathrm{nm})$}

e)

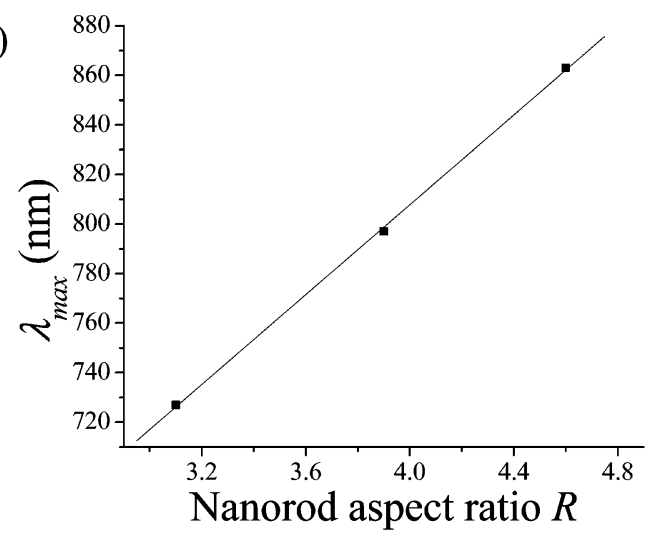

Figure 4. Tunability of the plasmon resonance maximum in nanoparticles. Variation of surface plasmon extinction maximum $\lambda_{\max }$ with (a) nanosphere diameter $D$, (b) nanoshell total radius $R_{2}$ at fixed $R_{1} / R_{2}=0.857$, (c) nanoshell core/shell ratio $R_{1} / R_{2}$ at fixed $R_{2}=70 \mathrm{~nm}$, (d) nanorod effective radius $r_{\text {eff }}$ at fixed aspect ratio $R=3.9$, and (e) nanorod aspect ratio $R$ at fixed $r_{\text {eff }}=11.43 \mathrm{~nm}$ (and straight line fit).

serve to provide additional wavelength tunability. ${ }^{57}$ Silica-gold nanoshells of various dimensions have been synthesized by Halas and co-workers, and such optical tunability has been experimentally demonstrated. ${ }^{35,57,59}$

In the case of nanorods, the plasmon resonance maximum (corresponding to the mode with the electric field parallel to the nanorod axis) can be shifted by either a change in size or aspect ratio. Figure $4 d$ shows the change in nanorod $\lambda_{\max }$ by changing the effective radius (hence volume) of the nanorod at a fixed aspect ratio. The linear dependence of $\lambda_{\max }$ on the nanorod aspect ratio $R\left(\lambda_{\max }=445.4+90.6 R\right)$ at a fixed effective radius is shown in Figure 4e. Similar dependence has been seen in experiments from Mohamed et al. ${ }^{87}$ and simulations for nanorods based on Gans theory. ${ }^{73}$ Gold nanorods can be synthesized by wet chemical ${ }^{88}$ as well as electrochemical methods ${ }^{89}$ in a range of aspect ratios from 2.0 to $18.0,{ }^{90}$ thus allowing for experimental tunability of the optical resonance wavelength. From the point of view of imaging applications, size tunability of the resonance wavelength in gold nanoparticles would allow multicolor labeling of different cell structures, similar to that allowed by quantum dots with size-dependent fluorescence. ${ }^{20,21}$

Dependence of Total Extinction Cross-section and Scattering/Absorption Ratio on Nanoparticle Dimensions. Two trends in the optical cross-sections with variation in nanoparticle size are uniform across different nanoparticle types. As seen in Figure 5a, $C_{\text {ext }}$ for nanospheres increases as the nanosphere size is increased. This trend has been commonly observed in experiments ${ }^{33,34}$ and reflects the direct dependence of the nanosphere extinction cross-section on the sphere volume in 

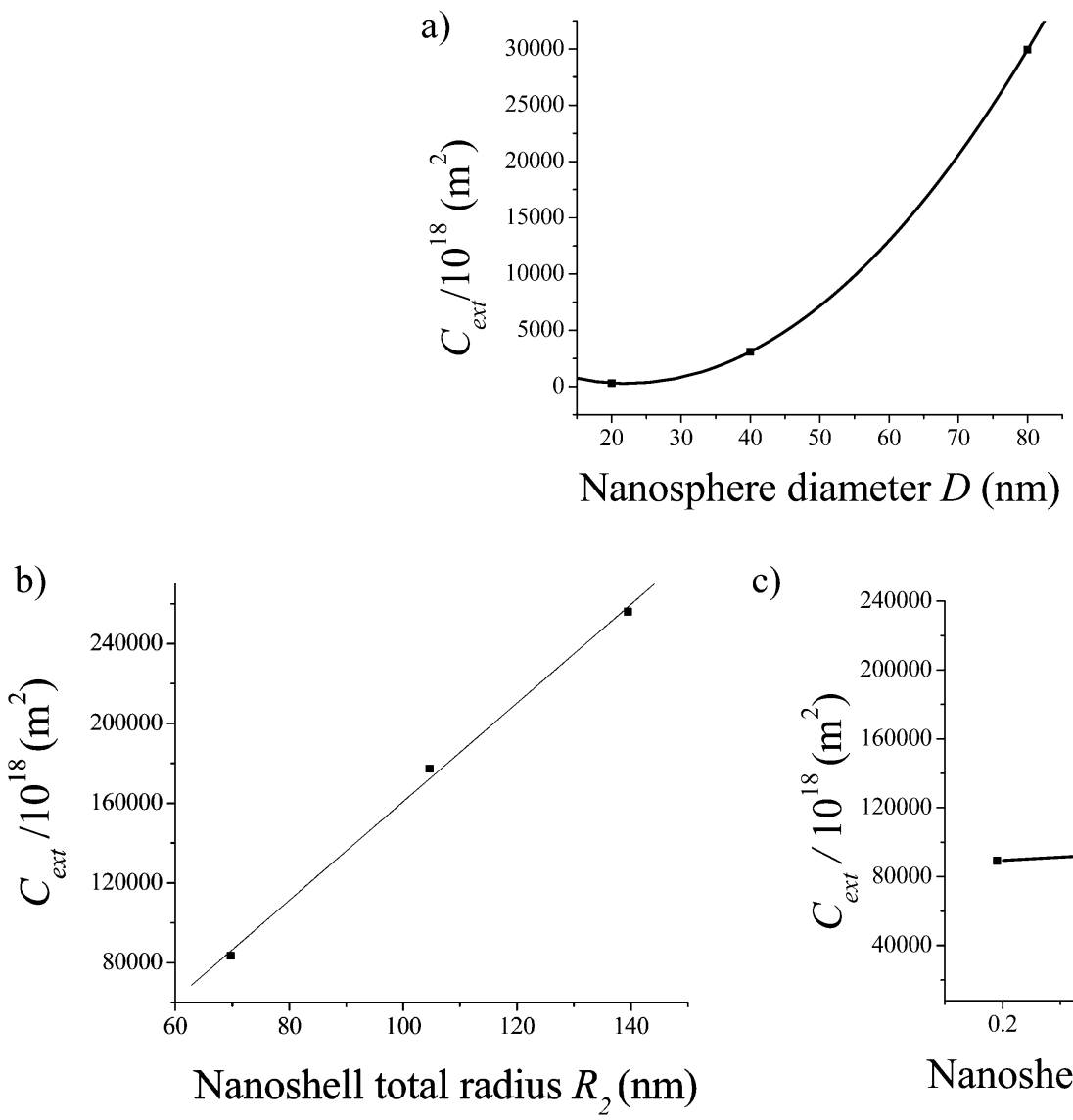

c)
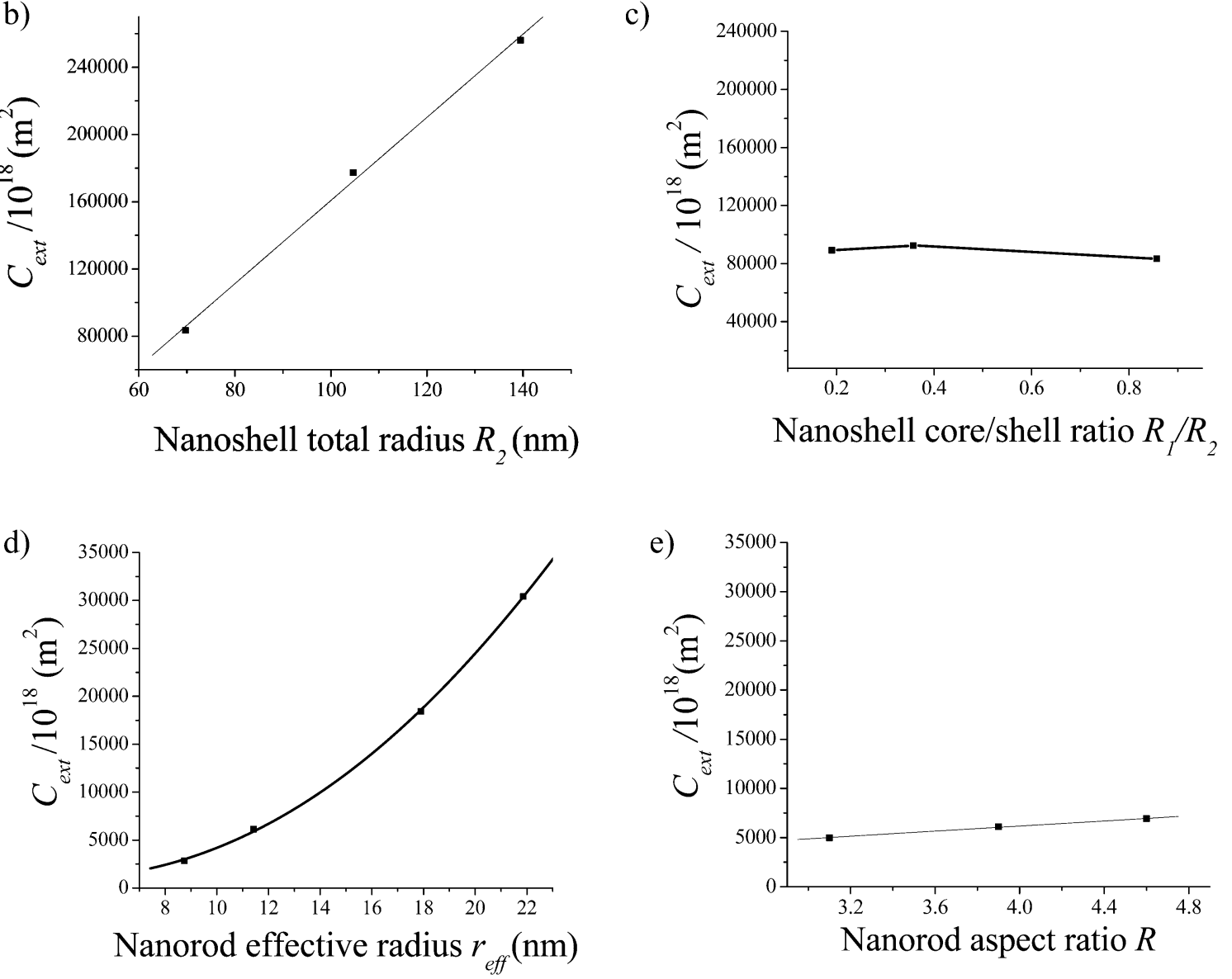

Figure 5. Tunability of the extinction cross-section of nanoparticles. Variation of $C_{\text {ext }}$ with (a) nanosphere diameter $D$, (b) nanoshell total radius $R_{2}$ at fixed $R_{1} / R_{2}=0.857$, (c) nanoshell core/shell ratio $R_{1} / R_{2}$ at fixed $R_{2}=70 \mathrm{~nm}$, (d) nanorod effective radius $r_{\text {eff }}$ at fixed aspect ratio $R=3.9$, and (e) nanorod aspect ratio $R$ at fixed $r_{\text {eff }}=11.43 \mathrm{~nm}$.

Mie theory (when the dipole mode contributes predominantly).,2,36 At the same time, with increasing nanosphere diameter, the relative contribution of scattering to the total extinction $C_{\mathrm{sca}} / C_{\mathrm{abs}}$ increases as seen from Figure 6a. The magnitude of the extinction increases linearly with the total nanoshell size $R_{2}$ as seen from the plot in Figure $5 \mathrm{~b}$ and rapidly with the effective radius $r_{\text {eff }}$ in the case of nanorods (Figure $5 d)$. The relative scattering contribution increases with increase in the total nanoshell size $R_{2}$ (Figure 6b) and the effective radius $r_{\text {eff }}$ in the case of nanorods (Figure 6d). The increase in the ratio of scattering to absorption with the nanoparticle volume has been related to increased radiative damping in larger nanoparticles based on experimental scattering spectra of gold nanospheres and nanorods measured by Sönnichsen et al. ${ }^{39,43}$ These trends suggest that larger nanoparticles would be more suitable for biological cell imaging applications based on light scattering, while those in the intermediate size range would serve as excellent photoabsorbers for laser photothermal therapy and applications based on absorption contrast. It can be seen that the change in the nanorod aspect ratio at a constant nanorod effective radius (or the nanorod volume) does not result in any considerable effect on either the extinction cross-section or the ratio of scattering to absorption (Figures $5 \mathrm{e}$ and $6 \mathrm{e}$ ). In the case of nanoshells, the relative core-shell dimensions do not have much effect on the magnitude of light extinction as long as the total nanoshell size (i.e., volume) is kept fixed (Figure 5c). However, a decrease in the core/shell ratio can be seen to be an effective handle in increasing the scattering contribution to the total extinction. For instance, a core-shell nanoparticle with the configuration $R_{1}=40 \mathrm{~nm} R_{2}=70 \mathrm{~nm}$ is dominantly 


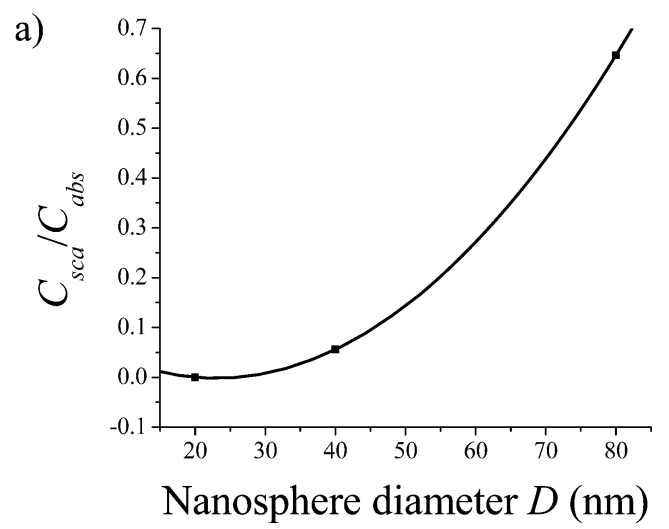

b)

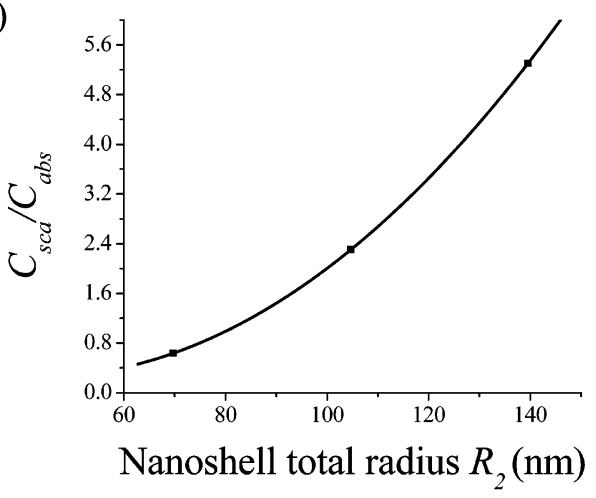

d)

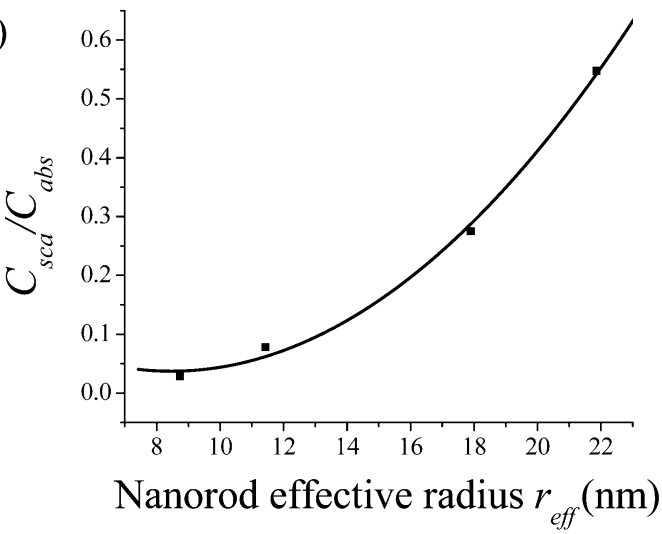

c)

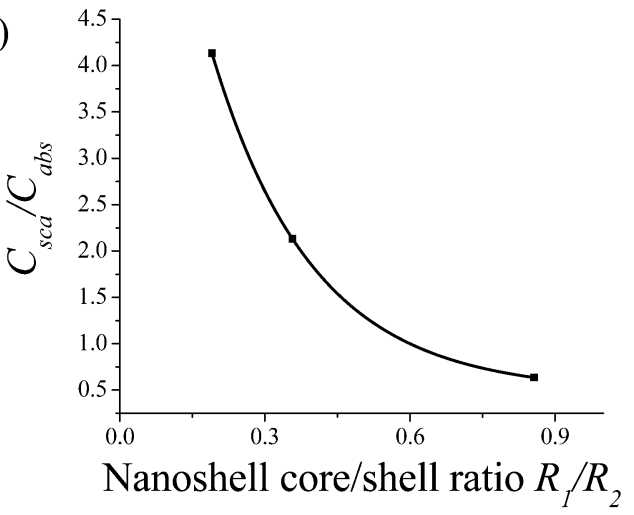

e)

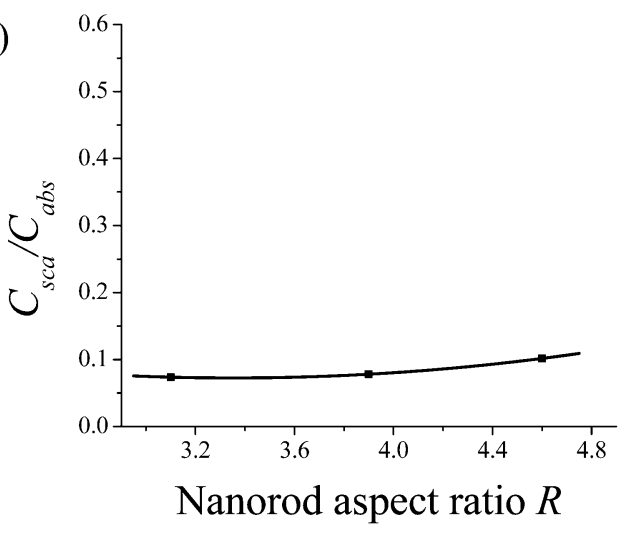

Figure 6. Tunability of the ratio of scattering to absorption of nanoparticles. Variation of $C_{\mathrm{sca}} / C_{\mathrm{abs}}$ with (a) nanosphere diameter $D$, (b) nanoshell total radius $R_{2}$ at fixed $R_{1} / R_{2}=0.857$, (c) nanoshell core/shell ratio $R_{1} / R_{2}$ at fixed $R_{2}=70 \mathrm{~nm}$, (d) nanorod effective radius $r_{\text {eff }}$ at fixed aspect ratio $R=3.9$, and (e) nanorod aspect ratio $R$ at fixed $r_{\text {eff }}=11.43 \mathrm{~nm}$.

scattering (Figure 2a), while a nanoshell with $R_{1}=60 \mathrm{~nm} R_{2}$ $=70 \mathrm{~nm}$ is a predominantly absorptive nanoparticle (Figure 2c). These trends in the nanoshell tunability have already been experimentally exploited by the Halas group for a dual cancer imaging and therapy approach. ${ }^{24,25,91}$

Size-Normalized Absorption and Scattering Cross-sections. The absolute magnitude of the optical cross-section does not provide a reliable measure for the optical properties of an ensemble of nanoparticles employed in real-life biomedical applications because smaller particles can be loaded in a given volume in greater numbers as compared to particles of a larger size. Therefore, a more meaningful property for comparison across a range of sizes is the size-normalized cross-section or volumetric coefficient $C / V$ where $V$ is the particle volume. ${ }^{36}$ The volumetric coefficients expressed in units of $\mu \mathrm{m}^{-1}$ give us the per micron absorption coefficient $\mu_{\mathrm{a}}$ and scattering coefficient $\mu_{\mathrm{s}}$ of the nanoparticles. Values of $\mu_{\mathrm{a}}$ and $\mu_{\mathrm{s}}$ have been tabulated (Table 1) for the different nanoparticle configurations. It can be seen from Table 1 that the nanoshell configuration used by Hirsch et al. and Loo et al. $\left(R_{1}=60 \mathrm{~nm}, R_{2}=70 \mathrm{~nm}\right)$ 24,25 offers a combination of high $\mu_{\mathrm{a}}\left(35.66 \mu \mathrm{m}^{-1}\right)$ and $\mu_{\mathrm{s}}(22.73$ $\mu \mathrm{m}^{-1}$ ) among all the different nanoshell configurations considered in our calculations, making them efficient agents for a dual imaging/therapy approach. The gold nanospheres have a higher $\mu_{a}$ than the nanoshells. Especially, the intermediate size nanospheres $(D=40 \mathrm{~nm})$ employed in the earlier photothermal therapy experiments from our laboratory ${ }^{26,27}$ offer the strongest photoabsorption with relatively low scattering among nanospheres. Larger nanospheres (e.g., $D=80 \mathrm{~nm}$ ) offer better scattering properties. Gold nanorods have $\mu_{\mathrm{a}}$ and $\mu_{\mathrm{s}}$ values that 
TABLE 1: Calculated Extinction Wavelength Maximum $\lambda_{\max }(\mathrm{nm})$, Per Micron Absorption Coefficient $\mu_{\mathrm{a}}$ (at absorption wavelength maximum $\lambda_{\mathrm{a}}$ ), Per Micron Scattering Coefficient $\mu_{\mathrm{s}}$ (at scattering maximum wavelength $\lambda_{\mathrm{s}}$ ), and Ratio of Scattering to Absorption Component of the Extinction $\mu_{\mathrm{s}} / \mu_{\mathrm{a}}$ for Gold Nanospheres, Gold Nanorods, and Silica-Gold Nanoshells of Different Dimensions

\begin{tabular}{|c|c|c|c|c|c|}
\hline nanoparticle type & dimensions & $\lambda_{\max } \mathrm{nm}$ & $\mu_{\mathrm{a}}\left(\lambda_{\mathrm{a}}\right) \mu \mathrm{m}^{-1}(\mathrm{~nm})$ & $\mu_{\mathrm{s}}\left(\lambda_{\mathrm{s}}\right) \mu \mathrm{m}^{-1}(\mathrm{~nm})$ & $\mu_{\mathrm{s}} / \mu_{\mathrm{a}}$ \\
\hline silica-gold nanoshells & $\begin{array}{l}R_{1}=40 \mathrm{~nm} \\
R_{2}=70 \mathrm{~nm}\end{array}$ & 843 & $50.61(843)$ & $4.31(843)$ & 0.09 \\
\hline silica-gold nanoshells & $\begin{array}{l}R_{1}=50 \mathrm{~nm} \\
R_{2}=70 \mathrm{~nm}\end{array}$ & 704 & $20.57(704)$ & $44.57(730)$ & 2.17 \\
\hline silica-gold nanoshells & $\begin{array}{l}R_{1}=60 \mathrm{~nm} \\
R_{2}=70 \mathrm{~nm}\end{array}$ & 892 & $35.66(892)$ & $22.73(892)$ & 0.64 \\
\hline silica-gold nanoshells & $\begin{array}{l}R_{1}=90 \mathrm{~nm} \\
R_{2}=105 \mathrm{~nm}\end{array}$ & 984 & $11.07(984)$ & $25.50(984)$ & 2.33 \\
\hline silica-gold nanoshells & $\begin{array}{l}R_{1}=120 \mathrm{~nm} \\
R_{2}=140 \mathrm{~nm}\end{array}$ & 1120 & $7.61(730)$ & $18.75(1120)$ & 2.44 \\
\hline silica-gold nanoshells & $\begin{array}{l}R_{1}=120 \mathrm{~nm} \\
R_{2}=155 \mathrm{~nm}\end{array}$ & 1160 & $7.26(582)$ & $15.44(1160)$ & 2.13 \\
\hline gold nanospheres & $D=20 \mathrm{~nm}$ & 521 & $73.72(521)$ & $0.45(535)$ & 0.01 \\
\hline gold nanospheres & $D=40 \mathrm{~nm}$ & 528 & $87.36(528)$ & $5.25(535)$ & 0.06 \\
\hline gold nanospheres & $D=80 \mathrm{~nm}$ & 549 & $67.88(549)$ & $45.94(560)$ & 0.68 \\
\hline gold nanorods & $\begin{array}{l}R=3.1 \\
r_{\text {eff }}=11.43 \mathrm{~nm}\end{array}$ & 727 & $741.86(727)$ & $54.70(727)$ & 0.07 \\
\hline gold nanorods & $\begin{array}{l}R=3.9 \\
r_{\text {eff }}=11.43 \mathrm{~nm}\end{array}$ & 797 & 907.09 (797) & $70.86(797)$ & 0.08 \\
\hline gold nanorods & $\begin{array}{l}R=4.6 \\
r_{\text {eff }}=11.43 \mathrm{~nm}\end{array}$ & 863 & $1003.87(863)$ & $102.05(863)$ & 0.10 \\
\hline gold nanorods & $\begin{array}{l}R=3.9 \\
r_{\mathrm{eff}}=8.74 \mathrm{~nm}\end{array}$ & 788 & $986.56(792)$ & $34.49(792)$ & 0.03 \\
\hline gold nanorods & $\begin{array}{l}R=3.9 \\
r_{\mathrm{eff}}=17.90 \mathrm{~nm}\end{array}$ & 815 & $601.47(817)$ & $172.32(817)$ & 0.29 \\
\hline gold nanorods & $\begin{array}{l}R=3.9 \\
r_{\mathrm{eff}}=21.86 \mathrm{~nm}\end{array}$ & 842 & $449.34(842)$ & $242.58(837)$ & 0.54 \\
\hline
\end{tabular}

are an order of magnitude higher than those of nanoshells and nanoparticles. While nanorods with a higher aspect ratio along with a smaller effective radius are the best photoabsorbers, the best scattering contrast for imaging is obtained from high-aspectratio nanorods with a larger effective radius or volume.

\section{Conclusion}

To obtain a quantitative guide for selection of nanoparticles for light-scattering and absorption-based applications in biomedicine, a systematic study of the trends in the optical resonance wavelength, the extinction cross-section, and the relative contribution of scattering to the extinction with changes in the nanoparticle dimensions, was undertaken for three different classes of nanoparticles viz. silica-gold nanoshells, gold nanospheres, and gold nanorods. It was clearly evident from the calculated spectra that the optical properties of nanoparticles were highly dependent on the nanoparticle size, shape, and core-shell composition. All three nanoparticle types had optical cross-sections a few orders of magnitude higher than those of conventional dyes. For all three nanoparticle types, the increase in the size resulted in an increase in the extinction crosssection as well as the relative contribution of scattering. However, nanoshells and nanorods were found more favorable for in vivo applications due to their tunable optical resonance in the NIR region. Moreover, their relative scattering to absorption contribution could be easily tuned by a change in their dimensions. For the comparison of the optical properties of nanoparticles across a range of sizes, size-normalized crosssections were calculated. From the numerical comparison, the gold nanorods are seen to offer the most superior NIR absorption and scattering at much smaller particle sizes. Smaller sized nanorods may also offer better cell uptake ${ }^{31}$ as compared to the larger nanoshells and nanospheres. This, in addition to the potential noncytotoxicity ${ }^{92}$ of the gold material, easy optical tunability, and facile synthesis, makes gold nanorods the most promising nanoparticle agents for use in biomedical imaging and photothermal therapy applications.

Acknowledgment. We thank Ivan Charamisinau for making available his program for Mie theory calculations of composite particles and B. T. Draine and P. J. Flatau for use of their DDA code, DDSCAT 6.1. This research was also partly supported by a grant (code no. 05K1501-02110) from the "Center for Nanostructured Materials Technology" under "21st Century Frontier R\&D Programs" of the Ministry of Science and Technology, Korea.

\section{References and Notes}

(1) McConnell, W. P.; Novak, J. P.; Brousseau, L. C., III; Fuierer, R. R.; Tenent, R. C.; Feldheim, D. L. J. Phys. Chem. B 2000, 104, 8925.

(2) Kreibig, U.; Vollmer, M. Optical Properties of Metal Clusters; Springer: New York, 1995; and references therein.

(3) Faraday, M. Philos. Trans. R. Soc. London 1857, 147, 145

(4) Burda, C.; Chen, X.; Narayanan, R.; El-Sayed, M. A. Chem. Rev. $\mathbf{2 0 0 5}, 105,1025$, and references therein.

(5) Katz, E.; Willner, I. Angew. Chem., Int. Ed. 2004, 43, 6042 and references therein.

(6) Mirkin, C. A. Inorg. Chem. 2000, 39, 2258.

(7) Pendry, J. B. Science 1999, 285, 1687.

(8) Maier, S. A.; Kik, P. G.; Atwater, H. A.; Meltzer, S.; Harel, E.; Koel, B. E.; Requicha, A. G. Nat. Mater. 2003, 2, 229-232.

(9) Riboh, J. C.; Haes, A. J.; McFarland, A. D.; Ranjit, C.; Van Duyne, R. P. J. Phys. Chem. B 2003, 107, 1772.

(10) Shafer-Peltier, K. E.; Haynes, C. L.; Glucksberg, M. R.; Van Duyne, R. P. J. Am. Chem. Soc. 2003, 125, 588.

(11) Storhoff, J. J.; Elghanian, R.; Mucic, R. C.; Mirkin, C. A.; Letsinger, R. L. J. Am. Chem. Soc. 1998, 120, 1959.

(12) Rosi, N. L.; Mirkin, C. A. Chem. Rev. 2005, 105, 1547 and references therein.

(13) Chen, J.; Wiley, B. J.; Li, Z.-Y.; Campbell, D.; Saeki, F.; Cang, H.; Leslie, A.; Lee, J.; Li, X.; Xia, Y. Adv. Mater. 2005, 17, 2255 and references therein.

(14) Yelin, D.; Oron, D.; Thiberge, S.; Moses, E.; Silberberg, Y. Opt. Express 2003, 11, 1385.

(15) Wang, Y.; Xie, X.; Wang, X.; Ku, G.; Gill, K. L.; O’Neal, D. P.; Stoics, G.; Wang, L. V. Nano Lett. 2004, 4, 1689. 
(16) Sokolov, K.; Follen, M.; Aaron, J.; Pavlova, I.; Malpica, A.; Lotan, R.; Richards-Kortum, R. Cancer Res. 2003, 63, 1999. 829.

(17) El-Sayed, I. H.; Huang, X., El-Sayed, M. A. Nano Lett. 2005, 5,

(18) Grainger, R. G. Br. J. Radiol. 1982, 55, 1

(19) Bugaj, J. E.; Achilefu, S.; Dorshow, R. B.; Rajagopalan, R. J. Biomed. Opt. 2001, 6, 122.

(20) Bruchez, M., Jr.; Moronne, M.; Gin, P.; Weiss, S.; Alivisatos, A. P. Science 1998, 281, 2013.

(21) Chan, W. C. W.; Nie, S. Science 1998, 281, 2016.

(22) Landsman, M. L. J.; Kwant, G.; Mook, G. A.; Zijlstra, W. G. J. Appl. Physiol. 1976, 40, 575.

(23) Hayat, M. A. Colloidal Gold: Principles, Methods and Applications; Academic Press: San Diego, 1989; Vol. 1.

(24) Hirsch, L. R.; Stafford, R. J.; Bankson, J. A.; Sershen, S. R.; Rivera, B.; Price, R. E.; Hazle, J. D.; Halas, N. J.; West, J. L. Proc. Natl. Acad. Sci. U.S.A. 2003, 100, 13549 .

(25) Loo, C.; Lowery, A.; Halas, N.; West, J.; Drezek, R. Nano Lett. $\mathbf{2 0 0 5}, 5,709$.

(26) El-Sayed, I. H.; Huang, X.; El-Sayed, M. A. Cancer Lett. 2005, in press, published online Sept 28, 2005, http://dx.doi.orgdoi:10.1016/j.canlet.2005.07.035

(27) Huang, X.; Jain, P. K.; El-Sayed, I. H.; El-Sayed, M. A. Photochem. Photobiol. 2006, in press, published online Jan 18, 2006.

(28) Huang, X.; El-Sayed, I. H.; Qian, W.; El-Sayed, M. A. J. Am. Chem. Soc. 2006, 128, 2115-2120.

(29) Aslan, K.; Lacowicz, J. R.; Geddes, C. R. Curr. Opin. Chem. Biol. $\mathbf{2 0 0 5}, 9,538$.

(30) Weissleder, R. Nat. Biotechnol. 2001, 19, 316.

(31) Zauner, W.; Farrow, N. A.; Haines, A. M. R. J. Control. Rel. 2001, 71,39

(32) Underwood, S.; Mulvaney, P. Langmuir 1994, 10, 3427.

(33) Link, S.; El-Sayed, M. A. J. Phys. Chem. B 1999, 103, 8410

(34) Link, S.; El-Sayed, M. A. Int. Rev. Phys. Chem. 2000, 19, 409.

(35) Oldenburg, S. J.; Averitt, R. D.; Westcott, S. L.; Halas, N. J. Chem. Phys. Lett. 1998, 288, 243.

(36) Bohren, C. F.; Huffman, D. R. Absorption and Scattering of Light by Small Particles; John Wiley: New York, 1983.

(37) Heilmann, A.; Kreibig, U. Eur. Phys. J.: Appl. Phys. 2000, 10, 193.

(38) Doremus, R. J. Chem. Phys. 1964, 40, 2389.

(39) Sonnichsen, C.; Franzl, T.; Wilk, T.; von Plessen, G.; Feldmann, J. New J. Phys. 2002, 4, 1.

(40) Scaffardi1, L. B.; Pellegri, N.; de Sanctis, O.; Tocho, J. O. Nanotechnology 2005, 16, 158 .

(41) Cini, M. J. Opt. Soc. Am. 1981, 71, 386

(42) Alvarez, M. M.; Khoury, J. T.; Schaaff, T. G.; Shafigullin, M. N.;

Vezmer, I.; Whetten, R. L. J. Phys. Chem. B 1997, 101, 3706.

(43) Sönnichsen, C.; Franzl, T.; Wilk, T.; von Plessen, G.; Feldmann,

J.; Wilson, O.; Mulvaney, P. Phys. Rev. Lett. 2002, 88, 077402.

(44) Millstone, J. E.; Park, S.; Shuford, K. L.; Qin, L.; Schatz, G. C.;

Mirkin, C. A. J. Am. Chem. Soc. 2005, 127, 5312

(45) Kreibig, U. J. Phys. F 1974, 4, 999.

(46) Doremus, R. J. Chem. Phys. 1964, 42, 414

(47) Pinchuk, A.; Hilger, A.; von Plessen, G.; Kreibig, U. Nanotechnology 2004, 15, 1890.

(48) Graff, A.; Wagner, D.; Ditlbacher, H.; Kreibig, U. Eur. Phys. J. D

$\mathbf{2 0 0 5}, 34,263$

(49) Jin, R.; Cao, Y.; Mirkin, C. A.; Kelly, K. L.; Schatz, G. C.; Zheng, J. G. Science 2001, 294, 1901.

(50) Pastoriza-Santos, I.; Marzán, L. M. Nano Lett. 2002, 2, 903.

(51) Chen, S.; Carroll, D. L. Nano Lett. 2002, 2, 1003.

(52) Sun, Y.; Mayers, B.; Xia, Y. Nano Lett. 2003, 3, 675

(53) Doremus, R.; Kao, S.; Garćıa, R. Appl. Opt. 1992, 31, 5773.

(54) Hubenthal, F.; Ziegler, T.; Hendrich, C.; Alschinger, M.; Träger,

F. Eur. Phys. J. D 2005, 34, 165

(55) Link, S.; Wang, Z. L.; El-Sayed, M. A. J. Phys. Chem. B 1999, 103,3529 4329.

(56) Liz-Marzán, L. M.; Giersig, M.; Mulvaney, P Langmuir 1996, 12

(57) Oldenburg, S. J.; Hale, G. D.; Jackson, J. B.; Halas, N. J. Appl. Phys. Lett. 1999, 75, 1063.

(58) Pastoriza-Santos, I.; Gomez, D.; Pérez-Juste, J. Liz-Marzá, L. M.; Mulvaney, P. Phys. Chem. Chem. Phys. 2004, 6, 5056.

(59) Oldenburg, S. J.; Jackson, J. B.; Westcott, S. L.; Halas, N. J. Appl. Phys. Lett. 1999, 75, 2897.

(60) Nehl, C. L.; Grady, N. K.; Goodrich, G. P.; Tam, F.; Halas, N. J.; Hafner, J. H. Nano Lett. 2004, 4, 2355. 714.

(62) Storhoff, J. J.; Lazarides, A. A.; Mucic, R. C.; Mirkin, C.; Letsinger,

R. L.; Schatz, G. C. J. Am. Chem. Soc. 2000, 122, 4640.

(63) Félidj, N.; Truong, S. L.; Aubard, J.; Lévi, G.; Krenn, J. R.; Hohenau, A.; Leitner, A.; Aussenegg, F. R. J. Chem. Phys. 2004, 120, 7141.

(64) Mie, G. Ann. Phys. (Leipzig) 1908, 25, 377.

(65) Draine, B. T.; Flatau, P. J. J. Opt. Soc. Am. A 1994, 11, 1491.

(66) Kelly, K. L.; Coronado, E.; Zhao, L. L.; Schatz, G. C. J. Phys.

Chem. B 2003, 107, 668 and references therein

(67) Schatz, G. C. J. Mol. Struct. 2001, 573, 73 and references therein.

(68) Lee, K. S.; El-Sayed, M. A. J. Phys. Chem. B 2005, 109, 20331

(69) Shuford, K. L.; Ratner, M. A.; Schatz, G. C. J. Chem. Phys. 2005, $123,114713$.

(70) Aden, A. L.; Kerker, M. J. Appl. Phys. 1951, 22, 1242.

(71) Link, S.; Mohamed, M. B.; El-Sayed, M. A. J. Phys. Chem. B 1999, 103, 3073 and correction; Link, S.; El-Sayed, M. A. J. Phys. Chem. B 2005, $109,10531$.

(72) Coronado, E. A.; Schatz, G. C. J. Phys. Chem. B 1999, 119, 3926

(73) Gans, R. Ann. Phys. (Leipzig) 1915, 47, 270.

(74) Charamisinau, I.; Happawana, G.; Evans, G.; Rosen, A.; Hsi, R. A.; Bour, D. Appl. Opt. 2005, 44, 5055 .

(75) Johnson, P. B.; Christy, R. W. Phys. Rev. B 1972, 6, 4370 4217

(77) Kreibig, U.; Genzel, L. Surf. Sci. 1985, 156, 678.

(78) The relationship between the extinction cross-section $\left(C_{\text {ext }}\right.$ in $\left.\mathrm{m}^{2}\right)$ and the molar extinction coefficient $\left(\epsilon\right.$ in $\left.\mathrm{M}^{-1} \mathrm{~cm}^{-1}\right)$ is expressed as $C_{\text {ext }}=$ $0.2303 \epsilon / N_{\mathrm{A}}$ where $N_{\mathrm{A}}$ is Avogadro's number.

(79) Urbanska, K.; Romanowska-Dixon, B.; Matuszak, Z.; Oszajca, J ; Nowak-Sliwinska, P.; Stochel, G. Acta Biochim. Pol. 2002, 43, 387.

(80) Chen W. R.; Adams R. L.; Carubelli, R.; Nordquist, R. E. Cancer Lett. 1997, 115, 25 .

(81) Chen, W. R.; Adams R. L.; Higgins A. K.; Bartels K. E.; Nordquist, R. E. Cancer Lett. 1996, 98, 169.

(82) Du, H.; Fuh, R. A.; Li, J.; Corkan, A.; Lindsey, J. S. Photochem. Photobiol. 1998, 68, 141

(83) Smithpeter, C.; Dunn, A.; Drezek, R.; Collier, T.; Richards-Kortum,

R. J. Biomed. Opt. 1998, 3, 429.

(84) Schenkman, K. A. Crit. Care Med. 2002, 30, 267.

(85) World Health Organization International Agency for Research on Cancer, http://www-cie.iarc.fr/monoeval/grlist.html.

(86) Aizpurua, J.; Hanarp, P.; Sutherland, D. S.; Käll, M.; Bryant, G. W.; Garciáde Abajo, F. J. Phys. Rev. Lett. 2003, 90, 057401.

(87) Mohamed, M. B.; Ismail, K. Z.; Link, S.; El-Sayed, M. A. J. Phys. Chem. B 1998, 102, 9370 .

(88) Murphy, C. J.; Jana, N. R. Adv. Mater. 2002, 14, 80-82.

(89) Yu, Y.; Chang, S.; Lee, C.; Wang, C. R. C. J. Phys. Chem. B 1997, 101,6661

(90) Jana, N. R.; Gearheart, L.; Murphy, C. J. J. Phys. Chem. B 2001, 105,4065

(91) Loo, C.; Lin, A.; Hirsch, L.; Lee, M.-H.; Barton, J.; Halas, N.;

West, J.; Drezek, R. Technol. Cancer Res. Treat. 2004, 3, 33.

(92) Connor, E. E.; Mwamuka, J.; Gole, A.; Murphy, C. J.; Wyatt, M. D. Small 2005, 1, 325-327. 